\title{
Creating reproducible thoracolumbar burst fractures in human specimens: an in vitro experiment
}

\author{
Ludwig Oberkircher, MD, Maya Schmuck, Martin Bergmann, MD, Philipp Lechler, MD, \\ Steffen Ruchholtz, MD, and Antonio Krüger, MD

\begin{abstract}
Department of Trauma, Hand and Reconstructive Surgery, Philipps University of Marburg, University Hospital Giessen and Marburg, Marburg, Germany
\end{abstract}

\begin{abstract}
OBJECT The treatment of traumatic burst fractures unaccompanied by neurological impairment remains controversial and ranges from conservative management to $360^{\circ}$ fusion. Because of the heterogeneity of fracture types, classification systems, and treatment options, comparative biomechanical studies might help to improve our knowledge. The aim of the current study was to create a standardized fracture model to investigate burst fractures in a multisegmental setting.

METHODS A total of 28 thoracolumbar fresh-frozen human cadaveric spines were used. The spines were dissected into segments (T11-L3). The T-11 and L-3 vertebral bodies were embedded in Technovit 3040 (cold-curing resin for surface testing and impressions). To simulate high energy, a metallic drop tower was designed. Stress risers were used to ensure comparable fractures. CT scans were acquired before and after fracture. All fractures were classified using the AO/OTA classification.
\end{abstract}

RESULTS The preparation and embedding of the spine segments worked well. No repositioning or second embedding of the specimen, even after fracture, was required. It was possible to create single burst fractures at the L-1 level in all 28 spine segments. Among the 28 fractures there were 16 incomplete burst fractures (Type A3.1), 8 burst-split fractures (Type A3.2), and 4 complete burst fractures (Type A3.3). The differences before and after fracture for stiffness and for anterior, posterior, and central heights were all significant $(p<0.05)$.

CONCLUSIONS The ability to create reproducible burst fractures of a single vertebral body in a thoracolumbar spine segment may serve as a basis for future biomechanical studies that will provide better understanding of mechanical properties or fixation techniques.

http://thejns.org/doi/abs/10.3171/2015.6.SPINE15176

KEY WORDS spine; fracture mechanism; burst fracture; fracture model; biomechanical; thoracolumbar; trauma

$\mathrm{B}$ URST fractures mostly occur in the thoracolumbar region and are one of the most common fracture types, especially in younger patients. ${ }^{2-4,13,15}$ Acute thoracolumbar burst fractures are classified according to the AO/OTA Classification ${ }^{12}$ as a subgroup of Type A fractures (A3). ${ }^{7}$ Burst fractures are defined by involvement of the posterior vertebral body wall. The height of the posterior vertebral wall is often reduced, and spinal canal compromise $^{11}$ often accompanies this compression fracture. Burst fractures can be subdivided into incomplete burst fractures (Type A3.1), burst-split fractures (Type A3.2), and complete burst fractures (Type A3.3). Per definition for all Type A fractures, the posterior ligament complex ${ }^{7}$ (PLC) has to remain intact. Treatment options for burst fractures without neurological symptoms discussed in the literature are controversial. Recommendations range from operative to conservative treatment..$^{6,8,11,16,19,21}$ However recent meta-analyses of the literature reveal no advantage for surgical treatment in comparison with conservative treatment. ${ }^{5,18}$ Various operative treatments are described in the literature: posterior stabilization with or without decompression, anterior fusion, $360^{\circ}$ fusion, and percutaneous instrumentation as well as cement augmentation or a combination of techniques..$^{19}$ Most recommendations and treatment options are based on biomechanical considerations, ${ }^{9}$ and clinical studies will continue to show advantages of different operative treatments. However, in vitro models are necessary to evaluate the stability of the reconstructed spine..$^{10}$ The most common challenge in designing biomechanical studies is the creation of a repro-

ABBREVIATIONS PLC $=$ posterior ligament complex.

SUBMITTED February 7, 2015. ACCEPTED June 30, 2015.

INCLUDE WHEN CITING Published online December 18, 2015; DOI: 10.3171/2015.6.SPINE15176. 
ducible burst fracture, especially if the vertebral bodies are not dissected. For example, to evaluate posterior stabilization, it is necessary to use a specimen that has at least 2 connected motion segments and create a burst fracture in the middle vertebral body. Several biomechanical studies have already established methods for creating thoracolumbar burst fractures in vitro; however, the number of specimens in these studies is usually small, or animal vertebrae were used instead of human specimens. , $^{7,10,20}$ The aim of the current study was to develop and standardize a method for an in vitro experiment to create reproducible thoracolumbar burst fractures in human spinal segments.

\section{Methods \\ Specimens}

A total of 28 fresh-frozen human cadaveric thoracolumbar spines were used (T11-L3). Only male donors (mean age at the time of death $64.9 \pm 6.5$ years) were selected based on anonymized patient profiles to reduce the risk of osteoporosis or poorer bone quality. The donors had no history of tumor, osteoporosis, arthritis, or use of medications that could lead to secondary osteoporosis. Prior to fracture generation, CT scanning of all spines was performed to identify any pathologies, especially preexisting vertebral fractures or deformities. All 28 specimens could be used. The spines were dissected into segments (T11-L3). Soft tissue was removed except for the intervertebral discs and the anterior as well as the posterior ligament complex. The vertebral bodies of T-11 and L-3 were stably embedded in Technovit 3040 (cold-curing resin for surface testing and impressions). Specimens were stored at $-20^{\circ} \mathrm{C}$ until testing. Just prior to the experiment, all specimens were thawed to room temperature.

\section{Fracture Generation}

To simulate a traumatic sudden impact we decided to use a high-speed trauma model, modified from the techniques described by Panjabi et al. and Kallemeier et al. ${ }^{10,14}$ To simulate high energy, a metallic drop tower with a height of $1.7 \mathrm{~m}$ was built, allowing a load of $7 \mathrm{~kg}$ to be dropped onto a horizontally aligned impounder. The design of the drop tower allows us to change the weight as well as the height. Impaction of the specimen was limited to $2.5 \mathrm{~cm}$ by simply blocking the track of the impactor (Fig. 1). To adjust for the different sizes of the specimens, the impounder was placed directly on top of the embedded specimen, and the drop tower was realigned for every specimen. To allow adjustment, the tube in which the weight fell could be moved up and down (Fig. 2). To simulate a model for further biomechanical evaluation, it was necessary to create the burst fracture in L-1 only. Therefore, stress risers were generated by cutting the superior endplates and laminae of L-1 prior to impact (Fig. 3).

\section{Fracture Classification}

All fractured spine segments were classified by 2 experienced spine surgeons (L.O. and A.K.) using CT scans. The AO/OTA Classification was used to classify the fracture types. ${ }^{12}$

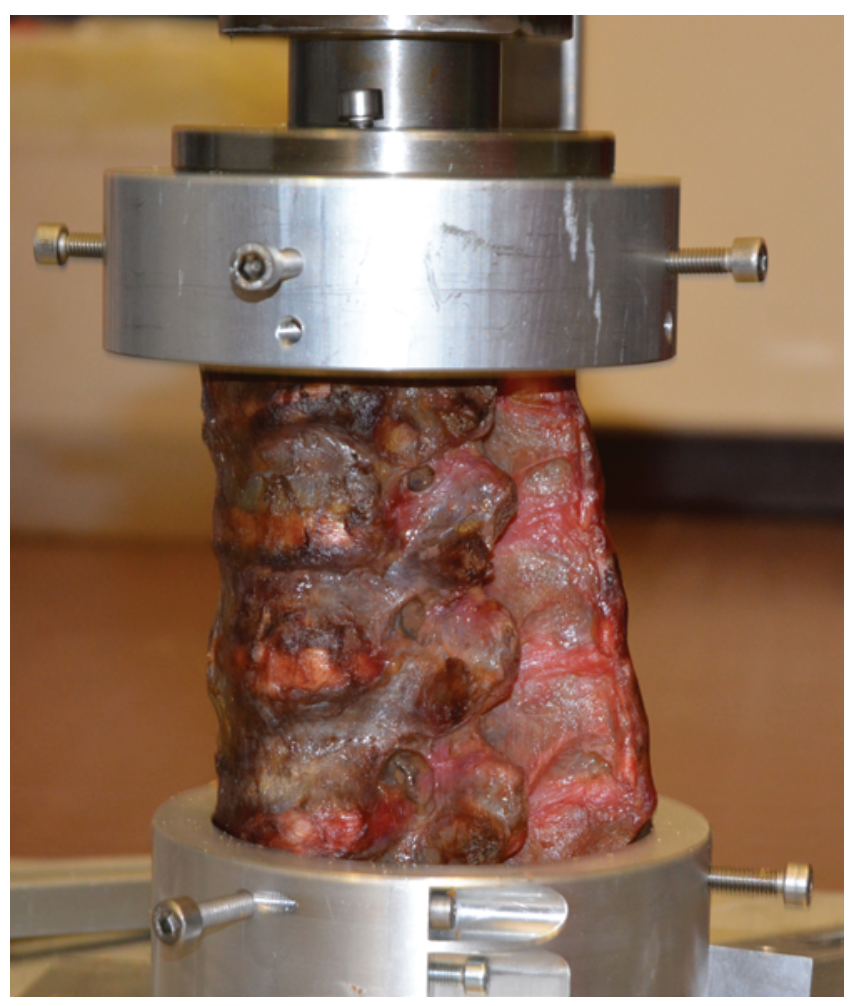

FIG. 1. Fixation of the specimen in the drop tower. Displacement was limited to $2.5 \mathrm{~cm}$ by simply blocking the track of the impactor. The impounder is placed directly on top of the embedded specimen (top of the picture). Figure is available in color online only.

\section{Stiffness Measuring}

To measure the instability of the created thoracolumbar burst fractures, stiffness was measured with a servohydraulic test bench (Bose Electroforce LM2 Test Bench). For testing deformation, the specimens were exposed to an axial load of $600 \mathrm{~N}$ (50 cycles, $1 \mathrm{~Hz}$ ) before and after fracturing.

\section{Measurements of Vertebral Height}

Before and after creating the burst fractures, measurements of the vertebral body height of L-1 were performed by analyzing the CT scans. Vertebral heights were measured at the anterior and posterior vertebral walls as well as in the center of the vertebral bodies in the midsagittal plane of the vertebral body.

\section{Cobb Angle and Spinal Canal Compression}

The Cobb angle was measured in the midsagittal plane on CT scans, and spinal canal compromise was measured by analyzing the CT scans in the axial plane. Spinal canal compromise was measured as a percentage of the difference between the surface area of the spinal canal before and after fracture. Surface area was measured at the point of maximum compromise.

\section{Statistical Analysis}

For all parameters determined, the results are expressed as means and ranges $\pm \mathrm{SD}$. The test of significance 


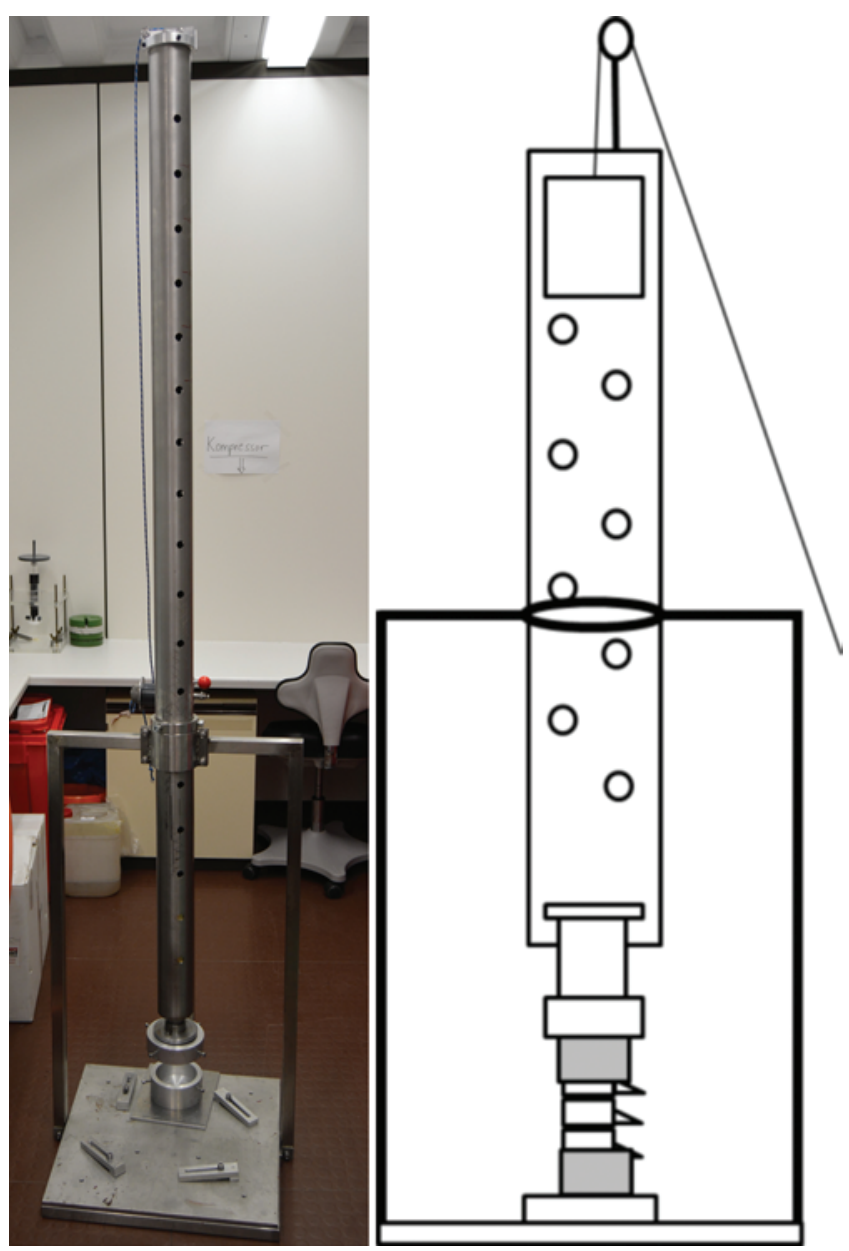

FIG. 2. Photograph (left) and schematic drawing (right) showing design of the drop tower. The tower is $1.7 \mathrm{~m}$ tall and allows different loads to be dropped onto a horizontally aligned impounder. The impounder is placed directly on top of the embedded specimen. Figure is available in color online only.

between results from study pairs was conducted by using the Student t-test and the Mann-Whitney test with significance $\mathrm{p}<0.05$.

\section{Results}

\section{Fracture Types}

The preparation and embedding of the spine segments worked well. No repositioning or renewal of the embedding was required, even after creation of the fracture. It was possible to create single burst fractures at the L-1 level in all 28 spine segments. A single drop of the weight was sufficient to create the fracture in all cases, and repetition was not necessary in any case. All fractures were classified according to the AO/OTA Classification. ${ }^{12}$ Basic characteristics of burst fractures include involvement of the posterior vertebral wall, reduction of the posterior height of the vertebral body, and some degree of narrowing of the spinal canal (Fig. 4).

Among the 28 fractures there were 16 incomplete burst fractures (A3.1), which were characterized by superior endplate destruction, depression in the anterior column,

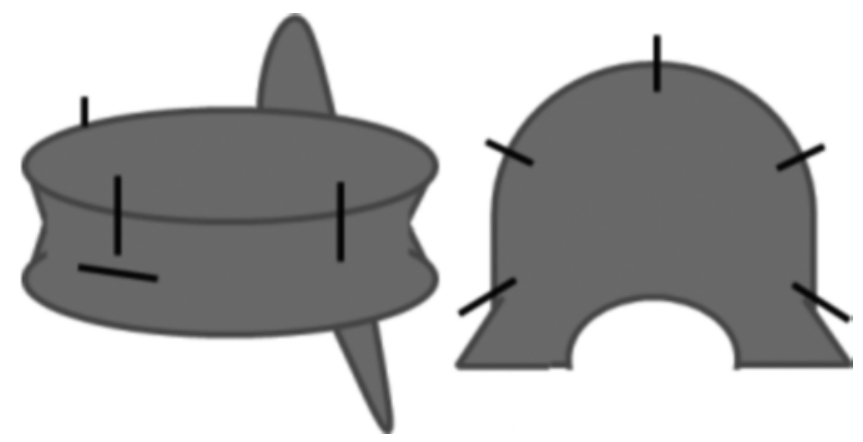

FIG. 3. To create reproducible burst fractures, surgical cuts were generated by cutting the superior endplate and lamina of L-1 prior to impaction.

and shattering of the middle column into 3-5 fragments, as well as the typical fragment retropulsed into the spinal canal. There were also 8 burst-split fractures (A3.2) and 4 complete burst fractures (A3.3) (Table 1).

\section{Stiffness}

The average stiffness before fracture was $728.5 \mathrm{~N} / \mathrm{mm}$ $( \pm 144.9)$. The average stiffness after fracture was $386.4 \mathrm{~N} /$ $\mathrm{mm}( \pm 84.6)$ (Table 1), and this difference was significant on the Student $t$-test $(\mathrm{p}<0.05)$.
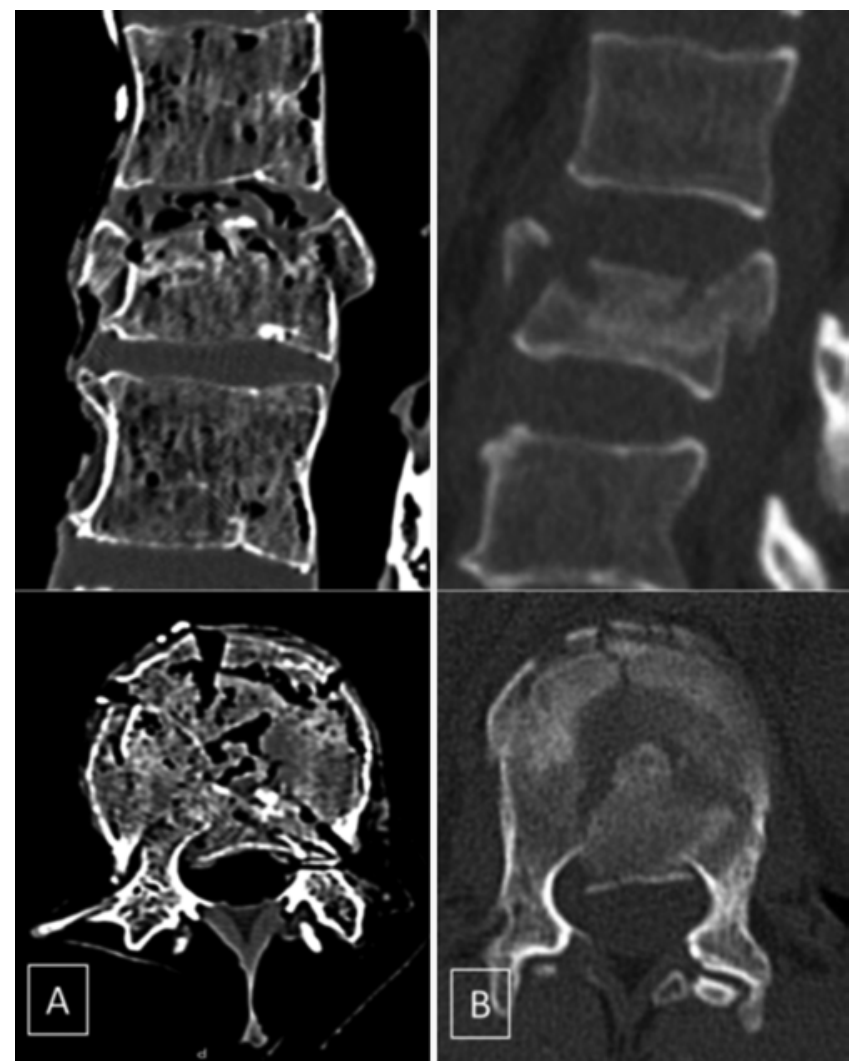

FIG. 4. Sagittal (upper) and axial (lower) CT reconstructions of an L-1 burst fracture created by the drop tower $(\mathrm{A})$ compared with one present in a representative patient (B). 
TABLE 1. Measurements and values for each fractured vertebral body specimen

\begin{tabular}{|c|c|c|c|c|c|c|c|c|c|c|}
\hline \multirow{2}{*}{$\begin{array}{l}\text { Specimen } \\
\text { No. }\end{array}$} & \multirow{2}{*}{$\begin{array}{l}\text { AO/OTA } \\
\text { Fx Type }\end{array}$} & \multicolumn{3}{|c|}{$\begin{array}{c}\text { Post-Fx Vertebral Body Height } \\
\text { ( } \% \text { of initial value) }\end{array}$} & \multicolumn{2}{|c|}{ Stiffness (N/mm) } & \multirow{2}{*}{$\begin{array}{c}\text { Post-Fx } \\
\text { Cobb } \\
\text { Angle }\left({ }^{\circ}\right)\end{array}$} & \multicolumn{2}{|c|}{$\begin{array}{l}\text { Area of Spinal Canal } \\
\qquad\left(\mathrm{mm}^{2}\right)\end{array}$} & \multirow{2}{*}{$\begin{array}{l}\text { Spinal Canal } \\
\text { Compromise (\%) }\end{array}$} \\
\hline & & Ant & Central & Pst & Pre-Fx & Post-Fx & & Pre-Fx & Post-Fx & \\
\hline 1 & A3.1 & 93.82 & 76.56 & 91.28 & 983.61 & 416.67 & 4.3 & 294 & 211 & 28.23 \\
\hline 2 & A3.2 & 88.71 & 72.59 & 84.29 & 666.67 & 422.54 & 3.6 & 270 & 222 & 17.78 \\
\hline 3 & A3.1 & 78.54 & 72.73 & 79.15 & 810.81 & 600.00 & 11 & 287 & 205 & 28.57 \\
\hline 4 & A3.3 & 89.80 & 71.60 & 85.57 & 1111.11 & 517.24 & 4.2 & 343 & 264 & 23.03 \\
\hline 5 & A3.1 & 80.93 & 94.38 & 91.78 & 612.24 & 273.97 & 15.8 & 316 & 255 & 19.30 \\
\hline 6 & A3.2 & 94.86 & 77.07 & 83.59 & 461.54 & 309.28 & 2.1 & 388 & 318 & 18.04 \\
\hline 7 & A3.2 & 93.84 & 70.94 & 91.86 & 869.57 & 540.54 & 15 & 344 & 330 & 4.07 \\
\hline 8 & A3.3 & 91.61 & 57.59 & 92.88 & 666.67 & 368.10 & 4 & 301 & 270 & 10.30 \\
\hline 9 & A3.3 & 88.76 & 64.31 & 89.55 & 625.00 & 295.57 & 6.9 & 267 & 220 & 17.60 \\
\hline 10 & A3.1 & 82.20 & 57.20 & 82.65 & 659.34 & 379.75 & 14.1 & 364 & 326 & 10.44 \\
\hline 11 & A3.2 & 94.42 & 74.31 & 88.29 & 674.16 & 384.62 & 4.6 & 317 & 225 & 29.02 \\
\hline 12 & A3.2 & 94.53 & 80.38 & 93.17 & 895.52 & 500.00 & 15.6 & 270 & 252 & 6.67 \\
\hline 13 & A3.2 & 89.63 & 70.09 & 87.59 & 714.29 & 350.88 & -3.3 & 301 & 230 & 23.59 \\
\hline 14 & A3.1 & 93.87 & 71.66 & 80.60 & 659.34 & 387.10 & 0.7 & 216 & 184 & 14.81 \\
\hline 15 & A3.1 & 79.56 & 66.93 & 78.05 & 810.81 & 416.67 & 3.1 & 305 & 216 & 29.18 \\
\hline 16 & A3.1 & 90.60 & 85.88 & 90.51 & 810.81 & 416.67 & 10.3 & 295 & 231 & 21.69 \\
\hline 17 & A3.1 & 86.89 & 81.27 & 90.24 & 779.22 & 434.78 & 6.8 & 372 & 298 & 19.89 \\
\hline 18 & A3.1 & 91.51 & 77.41 & 99.65 & 731.71 & 350.88 & 6.4 & 281 & 237 & 15.66 \\
\hline 19 & A3.3 & 94.83 & 56.67 & 81.97 & 530.97 & 320.86 & 7.9 & 365 & 355 & 2.74 \\
\hline 20 & A3.1 & 68.42 & 57.73 & 90.33 & 612.24 & 361.45 & -1.6 & 388 & 258 & 33.51 \\
\hline 21 & A3.1 & 97.38 & 84.96 & 85.96 & 722.89 & 379.75 & 17.4 & 338 & 276 & 18.34 \\
\hline 22 & A3.1 & 97.70 & 82.55 & 86.08 & 1000.00 & 434.78 & 7 & 292 & 275 & 5.82 \\
\hline 23 & A3.2 & 89.57 & 71.31 & 87.86 & 638.30 & 263.16 & 2.3 & 303 & 225 & 25.74 \\
\hline 24 & A3.1 & 85.57 & 75.49 & 88.27 & 750.00 & 408.16 & 5.4 & 298 & 218 & 26.85 \\
\hline 25 & A3.1 & 68.16 & 54.00 & 68.06 & 645.16 & 413.79 & 3 & 312 & 204 & 34.62 \\
\hline 26 & A3.2 & 83.94 & 50.00 & 82.02 & 606.06 & 272.73 & 4 & 335 & 275 & 17.91 \\
\hline 27 & A3.1 & 92.13 & 85.98 & 91.29 & 731.71 & 245.90 & 12.6 & 328 & 276 & 15.85 \\
\hline 28 & A3.1 & 89.26 & 77.61 & 89.90 & 618.56 & 352.94 & 7 & 279 & 208 & 25.45 \\
\hline Mean & & 88.3 & 72.1 & 86.9 & 728.5 & 386.4 & 6.3 & 313.2 & 252.3 & 19.5 \\
\hline SD & & 7.6 & 10.9 & 6.1 & 144.9 & 84.6 & 5.2 & 40.0 & 43.5 & 8.7 \\
\hline
\end{tabular}

Ant $=$ anterior $; \mathrm{Fx}=$ fracture; $\mathrm{pst}=$ posterior.

\section{Measurements of the Vertebral Heights}

After fracture the anterior, central, and posterior height of all L-1 vertebral bodies was significantly reduced. The average anterior height after fracture was $88.3 \%$ of initial prefracture height (range 68.2-97.7 [ \pm 7.6]), the average central height after fracture was $72.1 \%$ of initial prefracture height (range 50.0-94.4 [ \pm 10.9]), and the average posterior height after fracture was $86.9 \%$ of initial prefracture height (range 68.1-99.7 [ \pm 6.1]). All postfracture parameters were significant in comparison with prefracture values for vertebral body height when assessed by the Mann-Whitney test $(\mathrm{p}<0.05)$ (Table 1).

\section{Measurements of the Cobb Angle and Spinal Canal Compression}

The average Cobb angle after fracture was $6.3^{\circ}$ (range $-3.3^{\circ}$ to $\left.17.4^{\circ}\left[ \pm 5.2^{\circ}\right]\right)$. The average spinal canal surface area of the unfractured specimens was $313.2 \mathrm{~mm}^{2}( \pm 39.9$ $\left.\mathrm{mm}^{2}\right)$ and of the fractured vertebrae $252.3 \mathrm{~mm}^{2}( \pm 43.5$ $\mathrm{mm}^{2}$ ). The average spinal canal compression was $19.5 \%$ $( \pm 8.7 \%)$. These differences were significant as assessed by the Student t-test $(\mathrm{p}<0.05)$ (Table 1).

\section{Discussion}

Acute thoracolumbar burst fractures seem to be one of the most common thoracolumbar lesions, especially in young patients. ${ }^{2-4,13,15}$ Acute traumatic burst fractures are classified according to the AO/OTA Classification ${ }^{12}$ as a subgroup of Type A fractures (A3). ${ }^{7}$ They present with involvement of the posterior wall of the vertebral body and often with spinal canal compromise. ${ }^{11}$ Type A3 burst fractures are subdivided into incomplete burst fractures (A3.1), burst-split fractures (A3.2), and complete burst fractures (A3.3). An intact posterior ligament complex 
(PLC) is characteristic of all Type A fractures. The treatment of burst fractures without neurological deficit remains controversial. Recommendations range from operative to conservative treatment. ${ }^{6,8,11,16,19,21}$ However, previous reviews have shown no superior results for operative treatment in comparison with conservative treatment ${ }^{5,18}$ at an average follow-up of 4 years.

Various operative treatments are described in the literature: posterior stabilization with or without decompression, anterior fusion, $360^{\circ}$ fusion, and percutaneous instrumentation, as well as cement augmentation or a combination of techniques. ${ }^{17,19,22}$ In the US conservative treatment is often recommended if burst fractures are considered stable and if the PLC is intact. Treatment is guided by the Thoracolumbar Injury Classification and Severity (TLICS) score. In European countries burst fractures defined by involvement of the posterior wall of the vertebral body are surgically treated in most cases. These considerable differences in treatment underline the importance of understanding these fractures better.

Most recommendations for treatment are based on biomechanical considerations. ${ }^{9}$ Therefore, in vitro models in addition to clinical studies are necessary to evaluate the stability of the reconstructed spine. ${ }^{10}$ The creation of reproducible burst fractures seems to be the most common challenge for biomechanical studies, especially if the vertebral bodies were not dissected. For example, to evaluate posterior stabilization, it is necessary to have at least 2 connected motion segments and to create a burst fracture in the middle vertebral body without lesions in the upper and lower bodies. Several biomechanical studies have already established methods for creating thoracolumbar burst fracture in vitro; however, the numbers of specimens were small or animal specimens were used., ${ }^{7,10,20}$ Panjabi et al. described a high-speed trauma model in 1995. They used 16 human specimens, but did not indicate the level at which the fracture was created.$^{14}$ Kallemeier et al. used a model similar to that of Panjabi et al. for producing thoracolumbar burst fractures. They were able to produce burst fractures in every segment, but only 9 specimens were investigated. ${ }^{10}$ Jones et al. created burst fractures of L-1 in 5 cadaveric specimens, also using a free-fall protocol. ${ }^{9}$ Hartensuer et al. described a protocol using an Instron Testing System with an axial load of $300 \mathrm{~mm} / \mathrm{second}$ in 10 calf and 7 human specimens. ${ }^{7}$ In our study we decided to use a high-speed trauma model to simulate a traumatic sudden impact. Therefore, a modified system and protocol based on the ones used by Panjabi et al. and Kallemeier et al. was used. ${ }^{10,14}$ To ensure a burst fracture of L-1 only, stress risers were generated by cutting the superior endplate and lamina of L-1 prior to impaction, as described by Kallemeier et al. and Hartensuer et al., ${ }^{70}$ We were able to generate typical burst fractures in all 28 specimens (Fig. 2 ). Fractures appeared in the targeted L-1 without any lesions of the upper and lower vertebral bodies. We were mainly able to achieve incomplete burst fractures (Type A3.1), followed by burst-split fractures (Type A3.2), and complete burst fractures (Type A3.3). The latter group included only 4 cases. It is difficult to achieve consistent results when biological materials having different properties are used. A sudden impact is necessary for the creation of "traumatic" fractures. To compare different treatment methods, it is important to create a fractured vertebral body between unfractured vertebral bodies. We managed to create fractures at L-1 in every case. The distribution of fractures in our experimental series is comparable to the categories in the clinical series of burst fractures published by Altay et al. ${ }^{1}$ The distribution in their series was $61.9 \%$ for Type A3.1 fractures; $12.7 \%$ for Type A3.2 fractures; and $25.4 \%$ for Type A3.3 fractures. Similar percentages to these clinical values were obtained using our model.

In all fractures we were able to generate a significant loss of verterbral body height comparable to the results of Kallemeier et al. and Jones et al. ${ }^{9,10} \mathrm{Cobb}$ angles as well as spinal canal compression were comparable to the results of these two studies as well.,.10

Our study has some limitations. To create a single L-1 fracture, standard bone defects were necessary to create pre-stressing. Jones et al. criticized this type of model in their discussion. Their recommendation is a complete potting of the upper and lower vertebral bodies with rigid foam to get a single burst fracture. ${ }^{9}$ However, if further biomechanical investigation follows burst fracture generation, access to adjacent vertebral bodies is necessary, especially for testing posterior stabilization.

Despite these limitations this study presents, to the best of our knowledge, the largest number of cases of in vitro burst fracture generation in human fresh-frozen specimens. We created reproducible burst fractures of a single vertebral body in thoracolumbar spines. We were able to create burst fractures in all 28 specimens at a defined level (L-1) leaving the adjacent levels above and below intact, which makes the model very cost-effective. Our results may help substantiate those of previous studies and serve as a basis for further biomechanical studies. There are different ways in which this model can help us understand burst fractures better. Biomechanical studies on concomitant injuries (e.g., injuries to the PLC) would be possible. Most importantly, this model has the potential to evaluate different treatment strategies (e.g., anterior or posterior approaches and augmentation techniques) in a multilevel setting.

\section{Conclusions}

To our knowledge, this study is the first investigation to create a large number of burst fractures (AO/OTA Type A3) in multisegmental spine samples. The ability to create reproducible burst fractures of a single vertebral body in a thoracolumbar spine segment may serve as a basis for future biomechanical studies to develop and test treatment strategies for thoracolumbar burst fractures.

\section{References}

1. Altay M, Ozkurt B, Aktekin CN, Ozturk AM, Dogan O, Tabak AY: Treatment of unstable thoracolumbar junction burst fractures with short- or long-segment posterior fixation in Magerl Type A fractures. Eur Spine J 16:1145-1155, 2007

2. Dai LY, Yao WF, Cui YM, Zhou Q: Thoracolumbar fractures in patients with multiple injuries: diagnosis and treatment-a review of 147 cases. J Trauma 56:348-355, 2004

3. Denis F: The three column spine and its significance in the classification of acute thoracolumbar spinal injuries. Spine (Phila Pa 1976) 8:817-831, 1983 
4. Gertzbein SD: Scoliosis Research Society. Multicenter spine fracture study. Spine (Phila Pa 1976) 17:528-540, 1992

5. Gnanenthiran SR, Adie S, Harris IA: Nonoperative versus operative treatment for thoracolumbar burst fractures without neurologic deficit: a meta-analysis. Clin Orthop Relat Res 470:567-577, 2012

6. Ha KI, Han SH, Chung M, Yang BK, Youn GH: A clinical study of the natural remodeling of burst fractures of the lumbar spine. Clin Orthop Relat Res (323):210-214, 1996

7. Hartensuer R, Gasch A, Gehweiler D, Schanz S, Schulze M, Matuszewski L, et al: Experimentally induced incomplete burst fractures - a novel technique for calf and human specimens. BMC Musculoskelet Disord 13:45, 2012

8. Hitchon PW, Torner JC, Haddad SF, Follett KA: Management options in thoracolumbar burst fractures. Surg Neurol 49:619-627, 1998

9. Jones HL, Crawley AL, Noble PC, Schoenfeld AJ, Weiner BK: A novel method for the reproducible production of thoracolumbar burst fractures in human cadaveric specimens. Spine J 11:447-451, 2011

10. Kallemeier PM, Beaubien BP, Buttermann GR, Polga DJ, Wood KB: In vitro analysis of anterior and posterior fixation in an experimental unstable burst fracture model. J Spinal Disord Tech 21:216-224, 2008

11. Langrana NA, Harten RD RD, Lin DC, Reiter MF, Lee CK: Acute thoracolumbar burst fractures: a new view of loading mechanisms. Spine (Phila Pa 1976) 27:498-508, 2002

12. Magerl F, Aebi M, Gertzbein SD, Harms J, Nazarian S: A comprehensive classification of thoracic and lumbar injuries. Eur Spine J 3:184-201, 1994

13. McLain RF: Functional outcomes after surgery for spinal fractures: return to work and activity. Spine (Phila Pa 1976) 29:470-477, Z6, 2004

14. Panjabi MM, Oxland TR, Kifune M, Arand M, Wen L, Chen A: Validity of the three-column theory of thoracolumbar fractures. A biomechanic investigation. Spine (Phila Pa 1976) 20:1122-1127, 1995

15. Reinhold M, Knop C, Beisse R, Audigé L, Kandziora F, Pizanis A, et al: [Operative treatment of traumatic fractures of the thoracic and lumbar spinal column: Part III: Follow up data.] Unfallchirurg 112:294-316, 2009 (Ger)

16. Shen WJ, Shen YS: Nonsurgical treatment of three-column thoracolumbar junction burst fractures without neurologic deficit. Spine (Phila Pa 1976) 24:412-415, 1999

17. Siebenga J, Leferink VJ, Segers MJ, Elzinga MJ, Bakker FC, Haarman HJ, et al: Treatment of traumatic thoracolumbar spine fractures: a multicenter prospective randomized study of operative versus nonsurgical treatment. Spine (Phila Pa 1976) 31:2881-2890, 2006

18. Thomas KC, Bailey CS, Dvorak MF, Kwon B, Fisher C: Comparison of operative and nonoperative treatment for thoracolumbar burst fractures in patients without neurological deficit: a systematic review. J Neurosurg Spine 4:351-358, 2006

19. Verlaan JJ, Diekerhof CH, Buskens E, van der Tweel I, Verbout AJ, Dhert WJ, et al: Surgical treatment of traumatic fractures of the thoracic and lumbar spine: a systematic review of the literature on techniques, complications, and outcome. Spine (Phila Pa 1976) 29:803-814, 2004

20. Wang XY, Dai LY, Xu HZ, Chi YL: A comparison of single and incremental impact approaches for producing experimental thoracolumbar burst fractures. J Neurosurg Spine 7:199-204, 2007

21. Weinstein JN, Collalto P, Lehmann TR: Thoracolumbar "burst" fractures treated conservatively: a long-term followup. Spine (Phila Pa 1976) 13:33-38, 1988

22. Yue JJ, Sossan A, Selgrath C, Deutsch LS, Wilkens K, Testaiuti M, et al: The treatment of unstable thoracic spine fractures with transpedicular screw instrumentation: a 3-year consecutive series. Spine (Phila Pa 1976) 27:2782-2787, 2002

\section{Disclosures}

Dr. Krüger reports that he received clinical or research support for the study from VEXIM SaS.

\section{Author Contributions}

Conception and design: Oberkircher, Krüger. Acquisition of data: Oberkircher, Schmuck, Bergmann. Analysis and interpretation of data: Oberkircher, Schmuck, Lechler, Krüger. Drafting the article: Oberkircher, Schmuck, Krüger. Critically revising the article: all authors. Approved the final version of the manuscript on behalf of all authors: Oberkircher. Statistical analysis: Oberkircher, Schmuck, Krüger. Administrative/technical/material support: Bergmann, Ruchholtz. Study supervision: Oberkircher, Ruchholtz, Krüger.

\section{Correspondence}

Ludwig Oberkircher, University Hospital Giessen and Marburg, Baldingerstrasse, Marburg 35033, Germany. email: oberkirc@ med.uni-marburg.de. 